\title{
New Perspectives on the Hole Argument
}

\author{
Bryan W. Roberts ${ }^{1}$. James Owen Weatherall ${ }^{2}$
}

Received: 14 February 2020 / Accepted: 18 February 2020 / Published online: 18 March 2020

(c) Springer Science+Business Media, LLC, part of Springer Nature 2020

\begin{abstract}
This special issue of Foundations of Physics collects together articles representing some recent new perspectives on the hole argument in the history and philosophy of physics. Our task here is to introduce those new perspectives.
\end{abstract}

Keywords Hole argument · Preface · Editorial

\section{Introduction}

Few topics in the philosophy of physics have received more attention in the past forty years than Einstein's hole argument. The history of the subject is perhaps well-known, but worth repeating. In 1913, Einstein presented the hole argument in an effort to show that there could be no adequate "generally covariant" or diffeomorphism-invariant theory of gravity, instead advocating his erroneous Entwurf field equations. ${ }^{1}$ But by the end of 1915 , he had rejected the hole argument and the Entwurf theory, in part because he had found general relativity. Over the subsequent decades, the hole argument would reappear in the work of various groups on quantum gravity, usually together with a standard story: Einstein's 1913 blunder was a failure to realise that he had chosen a preferred coordinate system, and so he prematurely rejected general covariance. In the Entwurf theory the preferred coordinate system arose in the description of a Newtonian limit, and in the hole argument it arose in the explicit expression of the metric. ${ }^{2}$

\footnotetext{
1 The Entwurf theory was published by Einstein and Grossman [15]. Einstein formulated various versions of the hole argument in 1913-1914; for a discussion, see Norton ([26], §5).

2 See Giovanelli $[17,18]$ for further details on the long history of the point coincidence argument in quantum gravity. See Lanczos ([21], p. 13-14) for a classic expression of the 'standard story' of Einstein's supposed blunder.
}

James Owen Weatherall

weatherj@uci.edu

1 London School of Economics \& Political Science, Houghton Street, London WC2A 2AE, UK

2 University of California, Irvine, Irvine, CA 92697-5100, USA 
This simple perspective on the hole argument changed following a remarkable event in the history of science: the discovery of Einstein's notebook of scratchpad calculations during those crucial years. They were discovered by John D. Norton and John Statchel in the Einstein Archive at Princeton, miscatalogued as lecture notes from the University of Zurich. Working independently following this discovery, Stachel $[33]^{3}$ and Norton [26] argued that Einstein's Entwurf theory and hole argument were not trivial blunders, but the result of deeper metaphysical convictions according to which, "point events of the spacetime manifold are incorrectly thought of as individuated independently of the [metric] field itself" Norton ([26], 256). Meanwhile, John Earman was working on the bearing of Leibniz's thinking on a similar view, following some early thinking of Howard Stein. ${ }^{4}$ Soon after, Norton, working now in collaboration with John Earman, argued that Einstein's argument could be reconstructed in a way that was still relevant to contemporary debates in philosophy of physics, as a Leibniz-inspired reductio of a metaphysical conviction in the reality of spacetime points that they dubbed "manifold substantivalism" [14]. Since then, hundreds of papers have appeared responding to the Earman-Norton argument, and much of the subsequent literature in the foundations of general relativity and elsewhere in philosophy of physics has been shaped by these debates.

The Earman-Norton version of the argument may be put as follows. Let $(M, g)$ be a relativistic spacetime. Choose an open set $O$ with compact closure (the "hole"), assumed to be a proper subset of $M$. Let $d: M \rightarrow M$ be a diffeomorphism, which we assume to be the identity on $M / O$, and to differ from the identify somewhere within $O$. To make the point especially striking and to follow Einstein's original argument (though this is not essential), let us suppose $(M, g)$ is a solution to Einstein's equation for some stress-energy tensor $T$ that happens to vanish within $O$. Now consider the spacetime $\left(M, d^{*} g\right.$ ), where $d^{*}$ is the pullback along $d$. (We assume that $g$ and $d$ have been chosen so that there exists some point in $O$ at which $d^{*}(g) \neq g$.) It follows from the foregoing that this spacetime, too, is a solution to Einstein's equation, with source term $T$. We now have two spacetimes, $(M, g)$ and $\left(M, d^{*} g\right)$, with the same sources, which differ within $O$; but which, by virtue of being isomorphic, do not differ in any of their observable properties, even in principle.

Now suppose that one accepts "manifold substantivalism": that is, one believes that the manifold $M$ represents spacetime, independently of any of the fields defined on $M$. Then one is committed, it would seem, to the claim that $(M, g)$ and $\left(M, d^{*} g\right)$ represent different physical possibilities, corresponding to different assignments of metrical values to points within $O$; but also that these two possibilities are empirically indistinguishable from one another. It follows that no amount of information about the metric (and sources) outside of $O$ can be sufficient to determine the value of the metric at points within $O$. For instance, suppose that $\Sigma$ is a spatial slice "before" $O$ (in a spacetime with temporal orientation). No specification of initial data on $O$ can determine the subsequent evolution of $g$ within $O$. Thus the manifold substantivalist is committed to a radical form of indeterminism. Conversely, Earman

\footnotetext{
${ }^{3}$ Stachel's article was originally delivered as a talk in 1980, but only appeared in print in 1989.

${ }^{4}$ This latter part of the history, and especially Stein's contribution, is less-often told; see Weatherall [37].
} 
and Norton suggest this conclusion "can be easily escaped by just accepting Leibniz equivalence", which is the view that $(M, g)$ and $\left(M, d^{*} g\right)$ (and, indeed, all isometric spacetimes) represent the same physical situation. This, they argue, is tantamount to denying manifold substantivalism.

The early literature on the hole argument, following on the heels of the landmark Earman-Norton paper, focused on metaphysical issues. ${ }^{5}$ Is there a form of "substantivalism" that avoids indeterminism? Various authors defended versions of substantivalism-dubbed, with a distinctly perjorative flavor, "sophisticated substantivalism" by Belot and Earman [4], though this name has since been reclaimed by the sophisticates-according to which either (a) of the various isomorphic spacetimes generated by the hole argument and similar constructions, only one represents a "real" physical possibility (e.g. [10, 22]); or else (b) all of those isomorphic spacetimes represent a single possibility, which is nonetheless a possibility in which spacetime is a substance (e.g. [6, 19, 31]). By the end of the 1990s, there was a broad (albeit hardly universal) consensus that substantivalism remained defensible in light of the hole argument; but that it required greater subtlety than anyone would have expected before Earman and Norton published their argument, because the hole argument effectively excluded manifold substantivalism, allegedly the most natural (substantivalist, realist) interpretation of general relativity.

\section{The Hole Argument, Revisited}

In the past decade, two papers have appeared that have challenged this rough consensus, and which have led to renewed interest in the hole argument and related issues. The first, by Shamik Dasgupta, appeared in 2011. Dasgupta's principal contention was that sophisticated substantivalism, in its various guises, is too facile. The reason is that the hole argument, for instance as expressed above, concerns assumptions about modality and determinism; the sophisticated substantivalist responds to it by denying those assumptions. She then insists that without those assumptions, the hole argument does not go through, and the challenge to substantivalism fails. But, Dasgupta argues, to deny these assumptions is a "bare modal claim" that avoids a certain challenge without providing a positive account of what the metaphysics of spacetime substantivalism is meant to be. Moreover, denying the modal assumptions needed to run the hole argument does not avoid the central issue lurking in the background of hole-argument-style constructions, which is that the structure a substantivalist wishes to attribute to the world - a structure that includes, among other things, points of space/time that have some ontological status independent of matter-is superfluous within general relativity, as demonstrated by the existence of isomorphic spacetimes with the same qualitative properties, differing in how those qualitative properties are associated with those substantival points. Dasgupta concludes that no

\footnotetext{
${ }^{5}$ For recent reviews of the literature, see [27, 28].
} 
satisfactory substantivalist position has ever been articulated, much less defended, within general relativity.

The second article, by one of us (Weatherall), first appeared online in $2014 .^{6}$ Weatherall argues that the hole argument, as stated by Earman and Norton and in the ensuing literature, trades on a subtle mathematical error. The hole argument involves considering two isomorphic models of general relativity, which we will now call $(M, g)$ and $(N, h)$, where we suppose there exists some diffeomorphism $f: M \rightarrow N$ such that $f^{*}(h)=g$. One then makes two claims: first, that these two spacetimes are, in some sense, the same, because they are isomorphic; and second, that they are nonetheless different, because they make different assignments of metrical values to points. It is the first claim that supports the idea that whatever else is the case, there is no empirically discernible difference between these models, whereas it is the second claim that supports the idea that these models represent prima facie different possibilities. But, Weatherall argues, these claims are each true only relative to certain maps: the first claim is true relative to the isomorphism $f$; whereas the second is true only relative to some other map, which by construction is not an isomorphism. The hole argument then requires one to assert both claims together, even though there is no map relative to which both are true. ${ }^{7}$ The fact that this second map is necessary is obscured by the fact that in standard presentations of the Earman-Norton hole argument, the two spacetimes are written with the same symbol, " $M$ ", representing the manifold in both cases. This choice misleadingly invites one to compare the manifolds with the identity map on $M$, even when that is not the appropriate or salient standard of comparison.

Weatherall goes on to argue that what is truly at issue is what structure one should take a model of general relativity to have. On his view, one ought to understand models of general relativity to have the structure of a Lorentzian manifold, $(M, g)$, which is defined (like all mathematical objects) only up to isomorphism; whereas the hole argument apparently invokes further structure, namely, structure that permits one to distinguish between isomorphic Lorentzian manifolds. This extra structure represents the sorts of facts about spacetime points that the manifold substantivalist wants to attribute to the world. But, Weatherall argues, Lorentzian manifolds do not have enough structure to express those facts. And so the hole argument should really be viewed as an argument against a subtly different position from the one it is generally taken to refute. It is not successful, he claims, as an argument that reifying the structure of a model of general relativity leads to indeterminism; but it is effective as an argument against adding, to the standard formalism of general relativity, additional structure that would represent the sorts of facts that the manifold substantivalist wishes to attribute to the world. On this latter interpretation, the hole argument is compelling, though one might think that much simpler arguments, such as arguments from structural parsimony, would rule against manifold substantivalism, too.

Dasgupta and Weatherall are motivated by very different concerns, and their papers are targeted at somewhat different audiences. But there is a certain sympathy

\footnotetext{
6 It was accepted for publication in July 2015, but only appeared in print in 2018.

7 See, also, Shulman [32], who independently offers a nearly identical argument.
} 
between their positions. In both cases, they aim to shift the discussion away from analyses of the modal character of spacetime invited by the hole argument, and refocus on issues of what structure we should attribute to space and time and why. Still, there is a major disagreement between them, which concerns just how the models of general relativity relate to that structure. Are those models best understood as representing the structure that Dasgupta associates with "thick" substantivalism, where the basic entities are spacetime points with "primitive identity", as Dasgupta suggests? Or are they best understood as having the capacity to represent (only) a weaker structure, consisting of just those geometrical properties that are preserved by isomorphisms, as Weatherall would have it?

In the years since these papers appeared, a new literature on the hole argument has developed, with a focus shaped by the reframing of the issues offered by Dasgupta and Weatherall, respectively. The present special issue collects together a broad and representative, though hardly complete, sample of these papers. It originated as the proceedings of a 1-day workshop organized by one of us-Roberts-at the London School of Economics in July of 2016; several of the papers included here (those by Roberts, Fletcher, and Ladyman) were presented and discussed at that meeting. Other papers in the volume come from participants at the meeting (e.g., Weatherall and Dougherty), but were not presented there; and still others were solicited from philosophers who could not attend, but whose perspectives on these issues we felt were important to include. We believe that gathering these papers together in a single journal issue provides a valuable resource for scholars who wish to see the current state of the field. Not everything that might appropriately appear here does_-notable absences include the recent paper by Belot [3] arguing against the widely held view, presupposed in much of the new literature, that diffeomorphisms are necessarily "symmetries"; and as-yet unpublished work by Arledge and Rynasiewicz [1] and by Pooley and Read [29] criticizing Weatherall's position-but what does appear is nonetheless representative.

The papers collected here attend to three intertwined threads: issues of representation; issues of mathematical foundations; and issues of determinism.

\section{Issues of Representation}

Earman and Norton's hole argument was packaged together with a claim about representation, the now-famous principle of Leibniz Equivalence, which they characterised as the claim that, "diffeomorphic models represent the same physical situation" ([14], p.522).

The first thread of issues in this special issue concerns the meaning and correct usage of such representations: what can a mathematical object represent in the physical world, what can it not, and how is this related to the symmetries of that object? In his paper "On Representational Capacities, with an Application to General Relativity", Fletcher [16] develops an account of the representational capacities of mathematical structures-a concept that Weatherall invokes, but does not adequately develop. Fletcher explores how, on his account, the isomorphisms between mathematical models, including the automorphisms of those models, constrain 
those models' representational capacities. He ultimately defends a view where, in general, one should expect a many-to-many relationship between the models of a physical theory and the physical situations that may be represented by this theory, but with the constraint that isomorphic models should always be taken to have the same representational capacities. He then uses the machinery he has developed to offer a critical reply to Belot [3], whose paper is noted above.

In "Regarding 'Leibniz Equivalence'," Roberts [30] offers a new analysis of Leibniz equivalence, the doctrine that Earman and Norton [14] identify as the natural resolution of the hole argument. Earman and Norton define Leibniz equivalence as the view that isomorphic models of general relativity represent the same physical possibility. Roberts, by contrast, distinguishes two different ways in which one might interpret this position. One, which he calls "weak Leibniz equivalence", is the view that isomorphic models may represent the same possibility, if one allows the representational relation between the model and the world to vary. The second view, which he calls "strong Leibniz equivalence", is the view that isomorphic models represent the same possibility with respect to the same representation relation, understood here as a map from the underlying manifold $M$ of a relativistic spacetime to locations in space and time. He goes on to argue that weak Leibniz Equivalence is irrelevant to the hole argument, while strong Leibniz equivalence is strictly false. Roberts points out that the hole argument can still be seen as a more direct argument against substantivalism, independent of the status of Leibniz equivalence, and urges that attention be shifted away from the latter. Meanwhile, Roberts contends, authors like Weatherall and Fletcher conflate the weak and strong versions of the doctrine when claiming that once one attends carefully to mathematical practice, the hole argument is "blocked". A version of his critique appeared online in 2014.

Bradley and Weatherall [5], in their paper "On Representational Redundancy, Surplus Structure, and the Hole Argument," explore what it means to say that a physical theory exhibits "representational redundancy". They distinguish three different senses of "representational redundancy", and argue that each of these has importantly different consequences regarding, for instance, whether the models of a theory exhibiting such redundancy should be said to have "surplus structure". They proceed to apply these distinctions first to Yang-Mills theory, where they contrast their position with other ones recently defended by Weatherall [35] and (especially) Nguyen et al. [24], and then to the hole argument. They argue that the hole argument highlights the fact that general relativity has representational redundancy in one sense-but not a sense that signals surplus structure.

Finally, in his paper "General-Relativistic Covariance," Dewar [12] comes at a closely related set of issues-concerning the hole argument, symmetries, and structure-from a different direction. He develops an account of the "general covariance" often attributed to general relativity. His motivation is a tension that he identifies between the "dynamical view" of spacetime theories, as developed by Brown [9] and others, and Leibniz equivalence, as discussed by Earman and Norton. (We will not attempt here to resolve the question of whether Dewar's analysis runs aground on the distinction between weak and strong Leibniz equivalence drawn by Roberts.) The version of the dynamical view that Dewar considers is one in which the structure of spacetime should be precisely that which is invariant under the symmetry 
group of the dynamics governing matter. But as Dewar argues, while this recipe for identifying spacetime structure can be used to extract Minkowski spacetime from Maxwell's equations expressed in standard coordinates on $\mathbb{R}^{4}$, it is much more difficult to see how it is supposed to apply once we allow for curved spacetime, as in general relativity. He suggests, in particular, that the recipe would lead one to conclude that spacetime is represented by a bare manifold, much like the Earman-Norton manifold substantivalist does; and thus, that it is difficult for an advocate of the dynamical view to accept the standard response to the hole argument. To avoid this conclusion, he proposes that one should think of the diffeomorphism freedom of general relativity as corresponding to an "internal" symmetry of the theory, analogous to the internal symmetries of Yang-Mills theory.

\section{Issues of Mathematical Foundations}

A second theme reflected in this special issue concerns further discussion of whether the hole argument can be expressed using only the structure of a Lorentzian manifold, when one insists on modern 'constructive' foundations for mathematics. In their paper "The Hole Argument in Homotopy Type Theory," Ladyman and Presnell [20], drawing on an argument to the same effect by Shulman [32], contend that the hole argument cannot even be expressed within Homotopy Type Theory, as long as one understands a Lorentzian manifold as a dependent type (which, they argue, one should). Homotopy type theory provides an approach to the foundations of mathematics that is 'constructive' in the spirit of constructive set theory and intuitionistic logic [34]. It is often presented together with an axiom called 'Univalence', which its supporters characterise as expressing the claim that equivalent mathematical structures can be identified. This kind of claim is strikingly similar to the expression of Leibniz Equivalence introduced in Earman and Norton's hole argument, and so one might worry that Leibniz equivalence is "built in" to Homotopy Type Theory through this axiom. But, as Ladyman and Presnell clarify, the assessment of the hole argument in Homotopy Type Theory depends only on the structure of dependent types, and not on other, potentially controversial, parts of Homotopy Type Theory such as the Axiom of Univalence. In order to express the hole argument, they conclude, one would need to introduce a new structure to represent spacetime, using ordered pairs rather than dependent types.

Dougherty [13], too, considers the hole argument from the perspective of Homotopy Type Theory in his paper "The Hole Argument, take $n$," but he comes to a somewhat different conclusion. He contends that the arguments of Weatherall [36] (and Shulman [32]) show that proper attention to mathematical practice, at least as formalized within Homotopy Type Theory, does resolve what he calls the "verificationist dilemma" of the hole argument, which is that the substantivalist is apparently committed to observationally indistinguishable, but nonetheless distinct, states of affairs. (It is this dilemma that Dasgupta [11] takes to be a stronger argument against thick substantivalism than the modal version of the argument emphasized by Earman and Norton [14] and others.) But, Dougherty continues, Homotopy Type Theory does not resolve a second dilemma, which he calls the "indeterminism 
dilemma". To defend this, he makes a proposal concerning how to formulate "determinism" within Homotopy Type Theory, and then concludes that on this definition, general relativity as standardly formulated is indeterministic after all. He goes on to argue that the hole argument should thus motivate adopting a different formalism for general relativity-or rather, a different category of models of general relativity-than philosophers have generally supposed. He suggests that the category he endorses is a better reflection of how working mathematical physicists think about general relativity.

\section{Issues of Determinism}

Dougherty's contention that there are problems concerning the hole argument and determinism that persist, or even come into clearer focus, in light of the recent literature, touches on a third theme of the issue. How should one understand determinism in the context of the hole argument? The final two papers in the volume revisit, in light of more recent developments, arguments due to Melia [23], Belot [2], and Brighouse [7], concerning the proper notion of "determinism" in physics.

In her paper, "Confessions of a (Cheap) Sophisticated Substantivalist," Brighouse [8] emphasizes the tension between "sophisticated substantivalism" —as realized, in particular, by the "thin substantivalism" articulated by Dasgupta—and a particular kind of dynamical symmetry breaking. The classic example, here, is due to Wilson [38]. Consider a cylindrical tower, perfectly symmetric along its central axis, with a weight on top of it that is likewise perfectly symmetric about the axis of symmetry of the tower. Suppose, finally, that this tower-and-weight system exists in a universe where there is some force acting on the weight, directed downwards along the axis of symmetry of the tower, and that there is nothing else in the universe that could break the axial symmetry. We suppose the tower will buckle at a particular time, and that the angle, relative to the central axis of the tower, is not specified.

In how many different ways can the tower buckle? That is, are there distinct possible histories, differing only with regard to the direction of buckling, even though all such histories are, in a straightforward sense, isomorphic? ${ }^{8}$ Brighouse, following a long tradition, argues that there ought to be a meaningful sense in which this sort of symmetry breaking is an instance of real indeterminism: the column may buckle in many different directions. But, she contends, this intuition is not within the scope of what is describable according to thin substantivalism (and sophisticated substantivalism more generally) because the various putatively distinct histories of the cylinder do not differ in any of the "thin" facts that support differences between possible worlds on such accounts. One might take the moral to be that substantivalists' attempts to thread the needle between denying the indeterminism of the hole argument while maintaining the indeterminism of Wilson columns rest on bare modal

\footnotetext{
${ }^{8}$ We set aside the question of whether the buckling we countenance here is compatible with any known theory of physics.
} 
claims, whereas if one follows Dasgupta's suggestion that the substantivalist must give a more direct characterization of her position, the tension reappears.

Finally, in "The Hole Argument Against Everything," Joshua Norton [25] contends that there is something deeply wrong with the reasoning involved in the hole argument. Consider: what the hole argument is claimed to establish is that there are some (putative) facts accepted by the substantivalist—namely, facts about at what spacetime point certain events occur-that are not determined by general relativity; from this, Earman and Norton conclude that to accept those facts leads to the unacceptable conclusion that the universe is indetermistic, and thus we should deny them. But, Norton points out, there are arguably many putative facts that are not determined by general relativity. (As a provocative example, he considers the existence of souls.) And yet it seems absurd to claim that, because general relativity cannot determine such facts, we should therefore reject them. The reason is simply that general relativity does not bear on such matters. From this, Norton concludes that there is something faulty in the hole argument itself: it takes for granted that a physical theory must determine certain facts if those facts are to be meaningful. But, he argues, whether this is so surely depends on the scope of the theory. And so for the hole argument to go through, one needs to provide a prior argument that facts about spacetime points are the sort of thing that fall within the scope of the theory.

Here Norton's argument has a certain resonance with Weatherall's argument (see also Stachel [33]) that the substantivalist is committed to structure that goes beyond what is needed (or generally used) to formulate general relativity. But whereas Weatherall (and Dasgupta) argue that this means one should reject that structure on Occamist grounds, Norton's position is that if this structure is not necessary for, or determined by, the dynamics of general relativity, the question of whether it exists in the world should be understood to be outside the scope of that theory. In other words, Weatherall and Dasgupta rely on a premise that one might be motivated to reject, which is that one should understand the structure of spacetime to be precisely what is required for general relativity and no more.

Taken together, these papers draw attention to important and unresolved issues in the foundations of general relativity, and of physics more generally. Striking disagreements persist among the authors, and in all of the papers, new arguments are given that challenge views defended by others in the collection. We hope and expect that this collection will serve as a launching point for further research in this area.

\section{References}

1. Arledge, C., Rynasiewicz, R.: On some recent attempted non-metaphysical resolutions of the hole dilemma. Unpublished, http://philsci-archive.pitt.edu/16343/(2019)

2. Belot, G.: New work for counterpart theorists: determinism. Br. J. Philos. Sci. 46(2), 185-195 (1995)

3. Belot, G.: Fifty million elvis fans can't be wrong. Noûs 52(4): 946-981. http://philsci-archi ve.pitt.edu/12779/ (2017)

4. Belot, G., Earman, J.: Pre-socratic quantum gravity. In: Callender, C., Huggett, N. (eds.) Physics Meets Philosophy at the Planck Scale, pp. 213-255. Cambridge University Press, New York (1999) 
5. Bradley, C., Weatherall, J.O.: On representational redundancy, surplus structure, and the hole argument. Found. Phys. This volume. http://philsci-archive.pitt.edu/16837/ (2020)

6. Brighouse, C.: Spacetime and holes. PSA: Proceedings of the Biennial Meeting of the Philosophy of Science Association, Volume 1: Contributed Papers, pp. 117-125 (1994)

7. Brighouse, C.: Determinism and modality. Br. J. Philos. Sci. 48(4), 465-481 (1997)

8. Brighouse, C.: Confessions of a (cheap) sophisticated substantivalist. Found. Phys. (2020). https ://doi.org/10.1007/s10701-018-0228-2

9. Brown, H.: Physical Relativity. Oxford University Press, Oxford (2005)

10. Butterfield, J.: The hole truth. Br. J. Philos. Sci. 40(1), 1-28 (1989)

11. Dasgupta, S.: The bare necessities. Philos. Perspect. 25: 115-160. https://www.jstor.org/stabl e/41329465 (2011)

12. Dewar, N.: General-relativistic covariance. Found. Phys. (2020). https://doi.org/10.1007/s1070 1-019-00256-0

13. Dougherty, J.: The hole argument, take $n$. Found. Phys. (2020). https://doi.org/10.1007/s1070 1-019-00291-X

14. Earman, J., Norton, J.: What price spacetime substantivalism? The hole story. Br. J. Philos. Sci. 38(4), 515-525 (1987)

15. Einstein, A., Grossman, M.: Entwurf einer verallgemeinerten Relativitätstheorie und einer Theorie der Gravitation. Zeittschrift für Mathematik und Physik 62, 225-261 (1913)

16. Fletcher, S.C.: On representational capacities, with an application to general relativity. Found. Phys. (2020). https://doi.org/10.1007/s10701-018-0208-6

17. Giovanelli, M.: Erich Kretschmann as a proto-logical-empiricist: adventures and misadventures of the point-coincidence argument. Stud. Hist. Philos. Mod. Phys. 44(2), 115-134 (2013)

18. Giovanelli, M.: Nothing but Coincidences: The Point-Coincidence Argument and Einstein's Struggle with the Meaning of Coordinates in Physics. Unpublished manuscript. (2019) http:// philsci-archive.pitt.edu/16830/

19. Hoefer, C.: The metaphysics of space-time substantivalism. J. Philos. 93(1), 5-27 (1996)

20. Ladyman, J., Presnell, S.: The hole argument in homotopy type theory. Found. Phys. (2020). https://doi.org/10.1007/s10701-019-00293-9

21. Lanczos, C.: Einstein's path from special to general relativity. General Relativity: Papers in Honour of J.L. Synge. Clarendon Press, Oxford, pp. 5-19 (1972)

22. Maudlin, T.: The essence of spacetime. PSA: Proceedings of the Biennial Meeting of the Philosophy of Science Association, Volume 2: Symposia and Invited Papers, pp. 82-91 (1988)

23. Melia, J.: Holes, haecceitism and two conceptions of determinism. Br. J. Philos. Sci. 50(4), 639664 (1999)

24. Nguyen, J., Teh, N. J., Wells, L.: Why surplus structure is not superfluous. Br. J. Philos. Sci. Forthcoming. http://philsci-archive.pitt.edu/14166/ (2018)

25. Norton, J.: The hole argument against everything. Found. Phys. (2020). https://doi.org/10.1007/ s10701-019-00258-y

26. Norton, J.D.: How einstein found his field equations: 1912-1915. Hist. Stud. Phys. Sci. 14, 253316 (1984)

27. Norton, J.D.: The hole argument. In Zalta, E.N. (ed.) The Stanford Encyclopedia of Philosophy, Summer 2019 edn. http://plato.stanford.edu/archives/sum2019/entries/spacetime-holearg/ (2019)

28. Pooley, O.: Substantivalist and relationalist approaches to spacetime. In Batterman, R. (ed.) The Oxford Handbook of Philosophy of Physics, Oxford University Press, Oxford, pp. 522-586. http://philsci-archive.pitt.edu/9055/ (2013)

29. Pooley, O., Read, J.: Why we don't need any more papers about the hole argument. Unpublished Manuscript (2019)

30. Roberts, B.: Regarding 'Leibniz Equivalence'. Found. Phys. This volume, Open Access: https:// doi.org/10.1007/s10701-020-00325-9 and Preprint: http://philsci-archive.pitt.edu/11192/ (2020)

31. Rynasiewicz, R.: The lessons of the hole argument. Br. J. Philos. Sci. 45(2), 407-436 (1994)

32. Shulman, M.: Homotopy type theory: A synthetic approach to higher equalities. In Landry, E. (ed.) Categories for the Working Philosopher. Oxford University Press, Oxford, pp. 36-57. https ://arxiv.org/abs/1601.05035 (2017)

33. Stachel, J.: Einstein's search for general covariance, 1912-1915. In: Howard, D., Stachel, J. (eds.) Einstein and the History of General Relativity, pp. 62-100. Birkhauser, Boston (1989)

34. Univalent Foundations Program, T.: Homotopy Type Theory: Univalent Foundations of Mathematics. https://homotopytypetheory.org/book, Institute for Advanced Study (2013) 
35. Weatherall, J.O.: Understanding gauge. Philos. Sci. 83(5): 1039-1049. http://philsci-archive.pitt. edu/14198/ (2016)

36. Weatherall, J.O.: Regarding the 'hole argument'. Br. J. Philos. Sci. 69(2): 329-350. http://phils ci-archive.pitt.edu/11578/ (2018)

37. Weatherall, J.O.: Some philosophical prehistory of the (Earman-Norton) hole argument. Stud. Hist. Philos. Mod. Phys. http://philsci-archive.pitt.edu/15435/ (2020)

38. Wilson, M.: There's a Hole and a Bucket, Dear Leibniz. Midwest Stud. Philos. XVIII, 202-240 (1993)

Publisher's Note Springer Nature remains neutral with regard to jurisdictional claims in published maps and institutional affiliations. 